\title{
Recherches et mesures exécutées dans la zone du glacier de la Marmolada
}

\author{
Investigations and measurements \\ on the Marmolada glacier
}

\author{
PAR Dino TONINI, \\ pROFESSEUR A I'UNIVERSITE DE PADOUE, \\ DIRECTEUR DU SERYIGE DES ÉTUDES DE LA SOCIETA ADMIATICA DI ELETTRICITA, vENISE
}

\begin{abstract}
Recherches poursuivies depuis plusieurs années sur le glacier de la Marmolada, qui est le plus grand glacier des Dolomites orientales, par les soins de la Commission des Neiges du Comité Glaciologique Italien, de l'Institut de Géographie de l'Université de Padoue, de l'ANIDEL, de l'Institut de Physique de l'Unibersité de Ferrare et de la Società Adriatica di Elettricità, sur la morphologie du glacier, sa dynamique et les principaux éléments météorologiques de la zone. Mise en place d'un appareillage très étendu et disposé pour obtenir les meilleurs résultats possibles.
\end{abstract}

\begin{abstract}
Investigations of several years duration on the Marmolada glacier, the biggest in the eastern Dolomites by the combined efforts of the Snow Commission of the Itatian Glaciological Committee, the University of Padua Geographical Institnte, ANIDEL, the University of Ferrara Physics Institute and "Società Adriatica di Elettricita." The following were studied: the glacier's morphology, the forces governing its movemient and the main meteorological influences in the area. Installation of an extensive system of measurement, placed so as to obtain optimum results.
\end{abstract}

Le glacier de la Marmolada (fig. 1), le plus vaste de ceux des Dolomites, appartenant au type classique pyrénéen ou de cirque et qui avait, en 1888, une étendue de $500 \mathrm{hm}^{2}$, occupe actuellement (1960) une zone de $300 \mathrm{hm}^{2}$, ce qui revient à dire qu'il a subi, au cours d'un peu plus de soixante ans, une diminution d'environ $40 \%$. Ce recul imposant était déjà manifeste il y a quelques années lorsque la Société Adriatique d'Electricité entreprit, dans la zone, des recherches en vue de la construction d'un réservoir hydroèlectrique. Le glacier de la Marmolada, toutefois, bien connu à cause de sa situation, a été, en outre, le centre des combats entre italiens et autrichiens qui ont eu lieu au cours de la guerre de 1915-1918. Il est bon de rappeler à ce propos que les accès aux galeries de rocade, entièrement creusés dans la glace et ayant une couverture d'au moins $2 \mathrm{~m}$, sont depuis longtemps découverts, ce qui témoigne, d'une manière on ne peut plus significative, de la diminution de la couverture de glace.

La nécessité de connaître le comportement du glacier en tant que source principale des écoum lements qui alimentent le réservoir même, a entraîné l'établissement, dès 1954, c'est-à-dire dès le début des travaux pour la construction, dans la cuvette appelée Fedaia, au pied du 


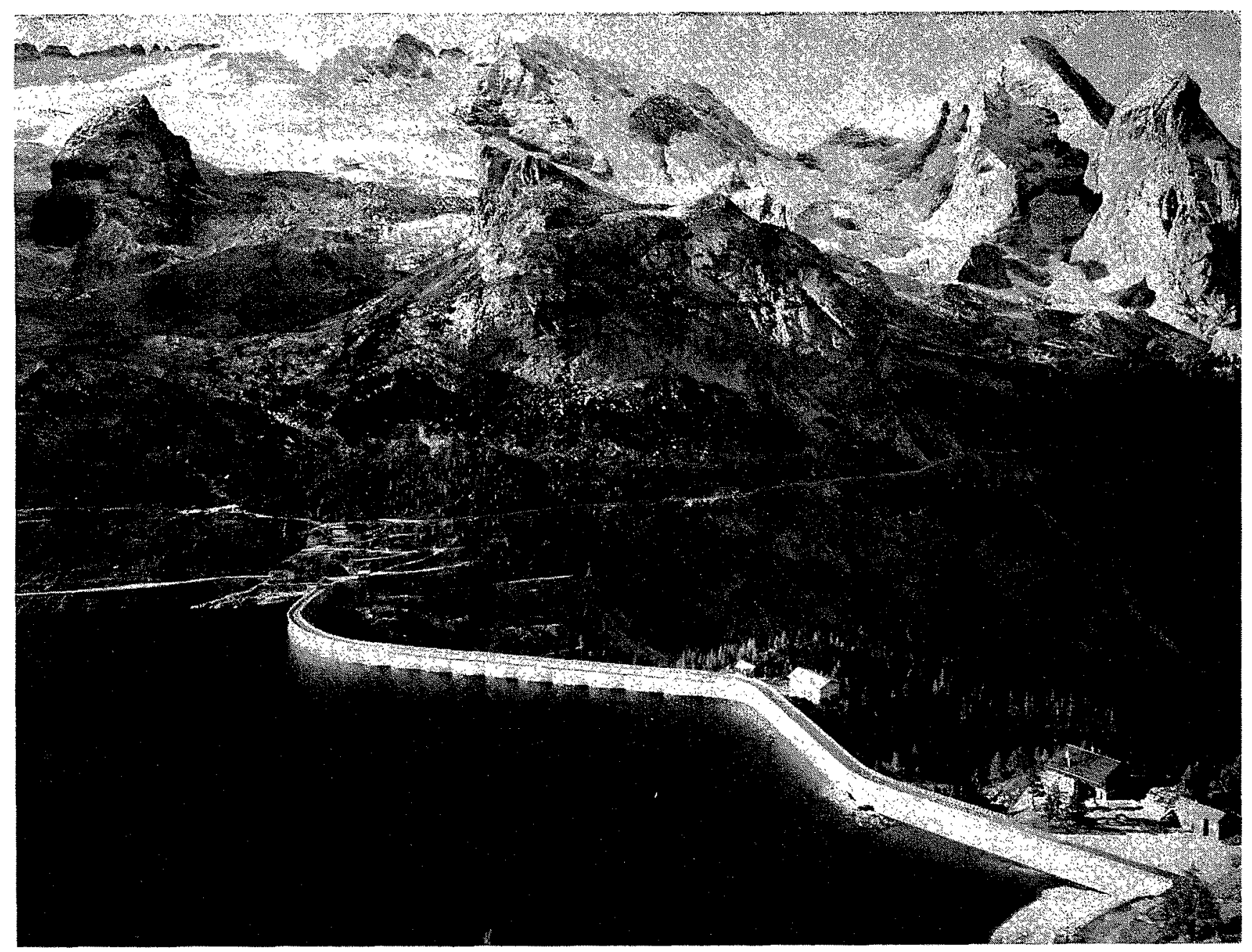

FIG. 1

Vue du glacier de Ia Marmolada et du barrage de Fedaia.

glacier, du réservoir terminé en 1956, d'un programme de recherches systématiques. Ces recherches coordonnées et réalisées sous les auspices de la Société Adriatique avec le concours de différentes institutions (ANIDEL, Comité Glaciologique Italien, Service Hydrographique de l'Etat, Institut de Géographie de l'Université de Padone, Institut de Physique de l'Université de Ferrare), ont abouti à la fondation d'un « Centre d'étude des Neiges et des Glaces» dont le siège est dans le bâtiment pour le personnel de garde du barrage.

Le réservoir de la Fedaia, situé à la cote 2053 , est formé d'un barrage à contreforts (fig. 1) haut de $65 \mathrm{~m}$ et long de $662 \mathrm{~m}$ et d'un barrage en terre situé à l'extrémité opposée. Le réservoir, dont la capacité d'accumulation est d'environ
$16 \mathrm{hm}^{3}$, alimente par chutes successives six usines dont la première utilise une hauteur de chute maximale de $583 \mathrm{~m}$.

On peut compter, pour la réalisation de ces recherches, sur une station météorologique installée près du barrage en béton et fonctionnant, pour la pluviométrie et pour la thermométrie, depuis 1937. Cette station a été agrandie par la suite et comprend actuellement les appareils suivants : pluviographe, nivometre, thermohydrographe, psychromètre, barographe, anémographe, pyrographe, héliophanographe. La station a été encore complétée par une station complémentaire installée dans le barrage en terre.

Suit ci-après la description des principales recherches en cours. 


\section{1. - MESURES DE LA DENSITE DE LA NEIGE}

A proximité du barrage de Fedaia, on a effectué, suivant des systèmes différents, plusieurs mesures visant à déterminer la consistance en eau de la couche de neige, c'est-à-dire la densité moyenne de la neige même.

On a commencé, au cours de l'hiver 19571958, par exécuter des mesures au moyen d'un type perfectionnè de pèse-neige construit par le Service Hydrographique du Magistrato alle Acque de Venise; ces mesures ont été répétées dans les saisons successives. Le système, suffisamment proche du type classique à prélèvement d'échantillon, reste toutefois extrêmement laborieux et ne saurait fournir que des données discontinues. C'est pourquoi, en collaboration avec l'Institut de Physique de l'Université de Ferrare, on a installé au même endroit, au cours de l'hiver 1957-1958, un dispositif pour la mesure de l'équivalent en eau de. la couche de neige au moyen de l'observation de l'absorption de rayons gamma.

L'installation comprend principalement une source de $\mathrm{Co}_{60}$ installée à l'extrémité d'un poteau haut de $4 \mathrm{~m}$, un détecteur Geiger-Müller installé au niveau du terrain et un compteur d'impulsions (scaler) situé dans une cabine, expressément aménagée à une distance de $30 \mathrm{~m}$ environ, où l'on a placé les appareils d'alimentation et de régulation, de même que quelques instruments de mesure.

La liaison entre les appareils situés à l'intérieur et les installation extérieures a été assurée par un câble à huit conducteurs muni d'un écran. La fréquence des impulsions émises par le détecteur Geiger a été mesurée quotidiennement au cours de l'hiver 1957-1958. En effet, d'après les variations de cette fréquence, on peut déterminer la densité de la

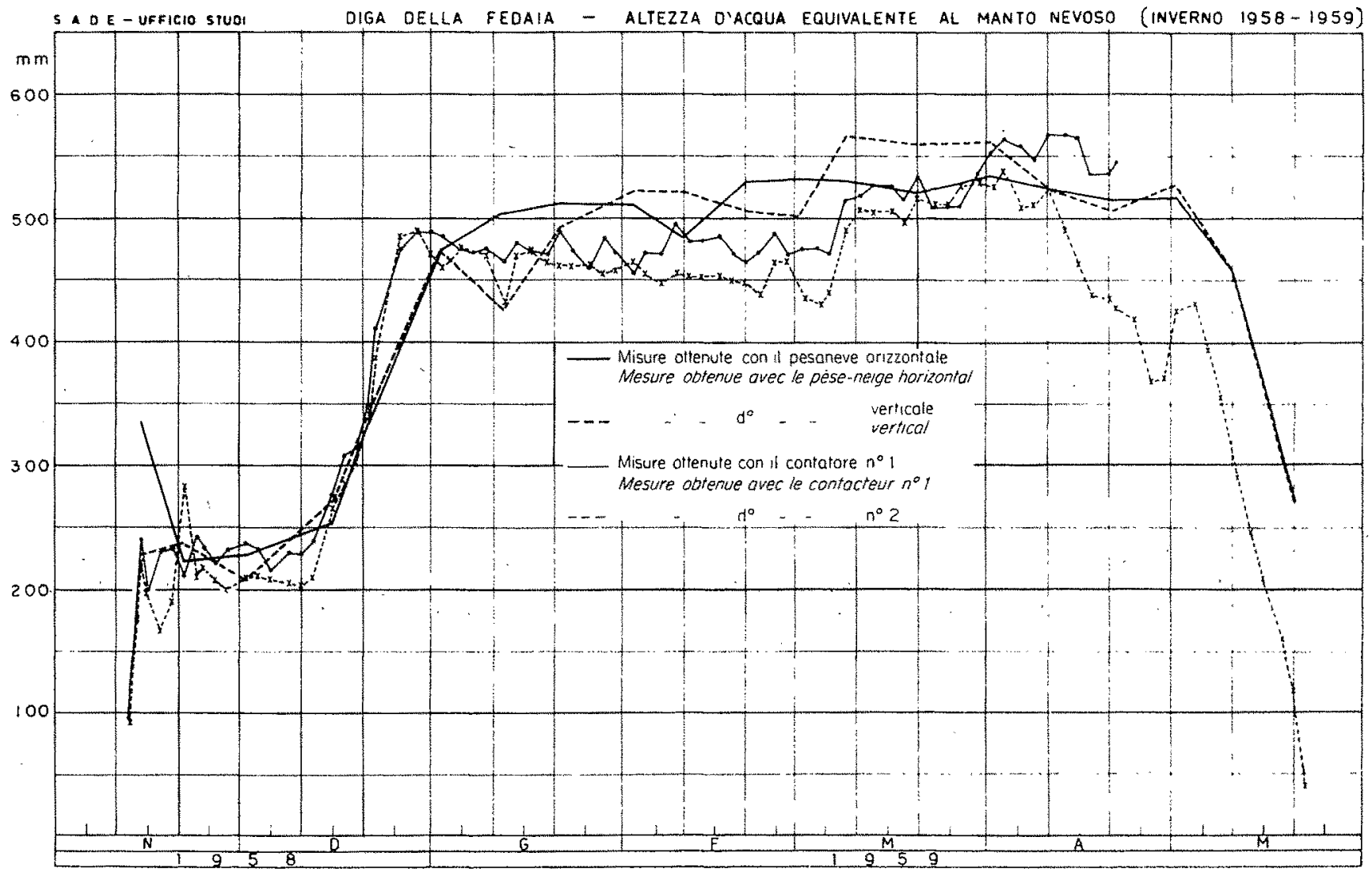

$0.63 / 5354$

Frg. 2

Diagramme de l'eau équivalant a la couche de neige dans la cuvette de Fedaia pendant l'hiver 1958-1959. 
couche neigeuse à l'aide de courbes d'étalonnage. Au début de l'hiver 1957-1958 on a calculé environ 1000 impulsions $/ \mathrm{mn}$ alors qu’à la fin de cette saison on n'a enregistré que 100 impulsions/mn. Il résulte de ces essais qu'il existait, à ce moment, une couche neigeuse équivalant à environ $50 \mathrm{~cm}$ d'eau. Pendant la période d'observation, le cours de la fréquence des impulsions marqua une augmentation graduelle de la masse de neige, avec de brusques variations en rapport avec les différentes précipitations.

Au cours de l'hiver $1958-1959$, on réalisa deux installations de ce type. L'une fut placée, comme l'année précédente, à proximité du barrage de Fedaia, l'autre près du barrage en terre à l'extrémité opposée du lac de Fedaia, dans l'endroit connu sous le nom de Maria al Lago. Le contrôle des deux installations, efrectué moyennant des mesures exécutées tous les dix jours avec deux pèse-neige, l'un horizontal et lautre vertical, a permis de constater un heureux accord entre les quatre séries de mesures différentes (fig. 2). A la fin de la saison, on détermina une couche neigeuse équivalant à environ $57 \mathrm{~cm}$ d'eau. Pendant toute la période d'observation, l'écart entre les données des quatre appareils demeura tonjours relativement modeste et, de toutes façons inférieur à $8 \mathrm{~cm}$ d'eau.

Des mesures analogues ont été répétées avec les mêmes appareils et dans les mêmes endroits, au cours des saisons successives. A la fin de l'hiver 1959-1960, on trouva que la couche neigeuse était équivalente à environ $65 \mathrm{~cm}$ d'eau, en avril 1961 , à $36 \mathrm{~cm}$ d'eau et, à la fin de l'hiver 1962 , on mesura $60 \mathrm{~cm}$ d'eau.

On a signalé quelques inconvénients dans l'emploi des appareils qu'on vient de décrire. Ils sont dus, pour la plupart, au câble de liaison reliant les instruments installés dans les cabines aux dispositifs extérieurs. Ces câbles présentaient un double inconvénient : celui tenant à la détérioration causée par des accidents extérieurs, et celui tenant du fait que, du moment qu'on ne saurait trop s'éloigner des installations d'alimentation, l'installation du câble finit par déterminer le choix de l'emplacement du point de mesure. Ơn a pourtant décidé de séparer complètement le détecteur Geiger des dispositifs de mesure et des sources d'alimentation, en radiotransmettant les impulsions à mesurer et en alitant, grâce à des batteries approprićes, le détecteur Geiger et le transmetteur. A la suite de nombreux essais et recherches, le Laboratoire de Recherches SADE a mis au point un dispositif pour la télétransmission des impulsions, dont on a construit un prototype en 1961. Ce modèle a été installé dans la localité de Maria al Lago, sous la source même qui alimente le susdit dispositif fonctionnant par câble.

Le schéma-type de la télémesure ne saurait être plus simple : le détecteur Geiger, alimenté à $810 \mathrm{~V}$, grâce à un système approprié de batteries, transmet les impulsions à un multivibrateur de Schmidt, qui les change de forme en les transférant sur une impédance plus basse. Dans le but d'empêcher qu'une succession trop rapide d'impulsions ne compromît le fonctionnement du système, on a relié la sortie du multivibrateur avec deux multivibrateurs de Eccles-Jordan (mieux connus sous le nom de Flip-Flop) dont l'effet combiné consiste en une division par quatre du nombre des impulsions. Le signal de sortie du deuxième Flip-Flop commande un relais télégraphique relié au transmetteur, lequel actionne un déclenchement toutes les quatre impulsions provenant du détecteur Geiger. Le transmetteur, du type commercial à quatre tubes électroniques, émet une onde portante ayant une fréquence de $114 \mathrm{MHz}$ que le relais télégraphique interrompt à chaque déclenchement.

La sortie du transmetteur est en communication directe avec l'antenne, au moyen d'un câble coaxial. Le dispositif est alimenté par trois accumulateurs au plomb du type employé dans les autos et ayant une capacilé totale de $90 \mathrm{~A} . \mathrm{h}$ (ampères-heure).

Dans le but d'éviter des pertes d'énergie dans les accumulateurs mêmes, on a prévu une commande à horlogerie quj fait fonctionner le dispositif une fois par jour. Une horloge mécanique à remontage électrique commande un microswitch qui coupe le circuit d'alimentation de tout le système en agissant aussi sur un relais qui est à son tour en série avec le circuit du détecteur Geiger. A la fin de la période de transmission (six minutes) on coupe la tension d'alimentation à tous les éléments et on ne laisse branchée que la modeste puissance nécessaire au remontage de l'horloge.

Lors la réception, le signal qui arrive sur l'antenne entre dans un convertisseur de fréquence 144-28 MHz et ensuite dans un récepteur de type professionnel à double conversion, qui réintègre les impulsions en alimentant un haut-parleur et un enregisteur à ruban magnétique. Il en résulte que le nombre des impulsions mesurées an moment de la réception est proportionnel au nombre des impulsions émises par le détecteur Geiger suivant le facteur $1 / 4$.

L'installation, bien qu'elle soit encore à sa phase expérimentale, fonctionne depuis quelques mois avec des résultats satisfaisants. 


\section{2. - MESURES DE LA TEMPERATURE DE LA NEIGE}

La température de la couche de neige est mesurée à l'aide d'une installation fixe de thermomètres électriques à résistance, placés à différentes hanteurs et à proximité du barrage de Fedaia. On sait que la température peut varier sensiblement si l'on procède du fond vers la surface de la couche de neige accumulée. Si l'on dispose d'une série verticale de thermomètres placés à une distance de $20 \mathrm{~cm}$ l'un de l'autre et enfoncés dans la couche neigeuse, on peut procéder à l'examen de ces vàriations avec la régularité nécessaire. Les thermomètres électriques permettent aussi d'effectuer un grand nombre de mesures à distance, dans des heures différentes du jour et dans toute l'épaisseur de la couche neigeuse, ce qui ne serait pas réalisable avec des thermomètres à mercure.

Cette station de mesure fonctionne régulièrement depuis l'hiver 1957-1958, après quelques tâtonnements initiaux pour obtenir l'emplacement satisfaisant des thermomètres, empêcher l'accumulation irrégulière de la neige et éliminer d'autres inconvénients susceptibles de diminuer la valeur de la signification des mesures mêmes. On a obtenu de meilleurs résultats en plaçant les différents thermomètres sur des supports indépendants, à différentes hauteurs du sol et en les disposant en hélice à diverses hauteurs.

Au centre de cette hélice, sur un support haut de $3 \mathrm{~m}$, on a installé un thermometre muni d'un écran approprié, destiné à mesurer la température de lair. Cette disposition nous a permis d'obtenir des séries de mesures assez complètes. Grâce à elles, on a pu entreprendre l'étude des variations thermiques, qui suivent des règles assez connues, du moins d'une manière générale, et établir des comparaisons utiles avec d'autres phénomènes météorologiques aussi bien qu'avec les transformations que la conche neigeuse subit au cours de chaque hiver.

Les résultats obtenus sont élaborés à l'Institut de Géographie de l'Université de Padoue. On a tracé des diagrammes qui indiquent les variations de la température dans la couche de neige en fonction de la profondeur. En élaborant ces diagrammes, on a pu obtenir des courbes qui représentent, en fonction du temps, la position des isothermes dans la couche de neige (fig. 3). La précision des mesures est assez satisfaisante (toutefois jusqu'à $1 / 4$ de degré). Il est encore dif-

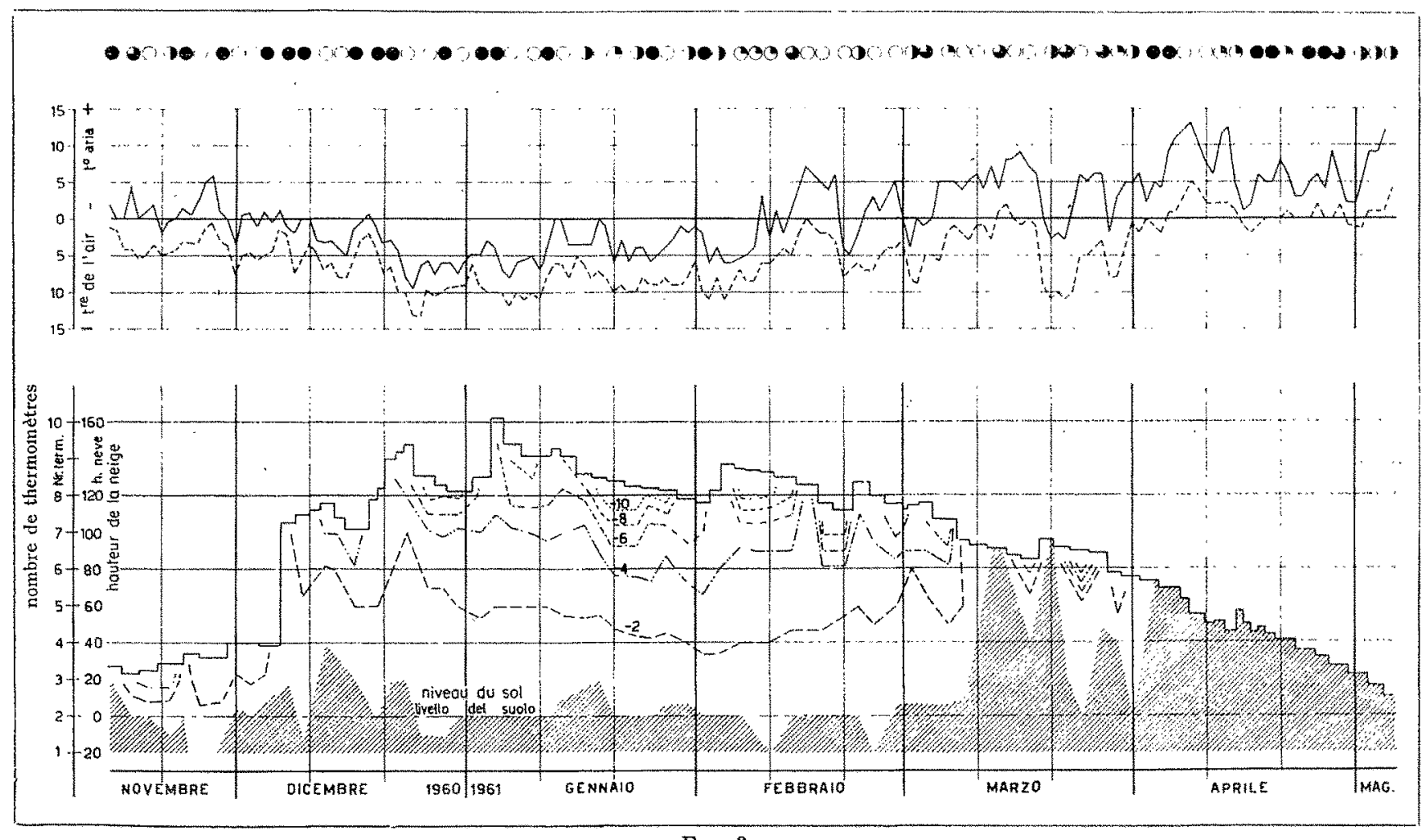

FIG. 3

Diagrammes de la température de l'air et courbes isothermes relevées dans la couche de neige pendant l'hiver 1960-1961. 
ficile de mesurer avec exactitude la température des zones les plus proches de la surface de la couche neigeuse, c'est-à-dire des premiers centimètres de l'épaisseur où, ainsi que l'on sait, on enregistre, dans les mois d'hiver, les tempéra- tures les plus basses. Il semble que, dans ce cas, on ne saurait éliminer la nécessité d'une mesure directe effectuée avec un thermomètre ou, de toute facon, avec un appareil mobile.

\section{3. - RECHERCHES SUR LA RADIATION TOTALE}

On sait que, suivant le schéma de Dines, le bilan de l'énergie radiante Soleil-atmosphèreTerre comprend différents éléments variables dans le temps et dans l'espace et interférents entre eux. Des 1,94 langley/mn (cal/ $\left.\mathrm{cm}^{2} \mathrm{mn}\right) \mathrm{de}$ la constante solaire à la limite de l'atmosphère, seule une quantité plus ou moins réduite suivant le degré de transparence de l'atmosphère même, atteint la surface de la Terre. Pendant la nuit, au contraire, toujours à condition qu'il y ait une transparence parfaite de l'atmosphère, la Terre pourrait irradier vers l'espace, d'après les calculs de M. le Professeur Zanetti de l'Université de Padoue, 0,45 langley/mn, $1 / 4$ environ de l'énergie maximale que la Terre recevrait sous forme de radiation solaire. Des conditions particulières (ciel bouché) peuvent annuler cette énergie radiante émise par la Terre sans que cela annule l'énergie irradiée ou réflexe de l'atmosphère et vers lespace et vers la Terre même.

Pour mesurer la différence $\Delta$, entre l'énergie irradiée par l'atmosphère vers la Terre et l'énergie émise par la Terre vers l'atmosphère. M. Zanetti a créé un appareil simple appelé frigorimètre, dont les mesures sont valables surtout dans les périodes au cours desquelles on n'a pas d'énergie irradiée par le Soleil vers la Terre, c'est-àdire, pour ce qui concerne l'application à la glaciologie, pendant la nuit ou pour les glaciers qui sont à l'ombre pendant le jour et pour lesquels de toutes façons la quantité de radiation solaire directe est insignifiante.

La différence $\Delta$ doit être considérée, surtout à des altitudes élevées, comme n'étant pas en rapport avec la température de l'air. En effet, la température de l'air est moins la cause qui influe par contact sur la température de la glace que l'indice du refroidissement subi par le glacier à la suite de l'irradiation vers l'espace. Cela revient à dire que la diminution de la température de l'air est provoquée par le refroidissement progressif du glacier résultant du solde négatif $\Delta$ du bilan radiant de l'atmosphère.

On devrait chercher une confirmation de ces prémisses d'ordre théorique dans le fait qu'on aurait un arrêt dans le processus d'ablation même avec une température de l'atmosphère supérieure à $0^{\circ} \mathrm{C}$ au cas où la valeur du bilan radiant de l'atmosphère serait négative. Le frigorimètre de M. Zanetti est formé de deux sphères, dont l'une est polie et l'autre noire, qu'on maintient constamment à une température de $36,5^{\circ} \mathrm{C}$. A l'intérieur des deux sphères, sont placées deux résistances et un thermostat qu'alimente un câble. L'autre bout du câble est relié au réseau à basse tension de la cabine de commande du barrage, où sont installés deux compteurs qui marquent la consommation d'énergie des deux sphères.

M. Zanetti obtient la valeur de $\Delta$ en langley/ min grâce à la formule :

$$
\Delta=\frac{q_{n}-1,07 \delta}{36,5-t_{\mathrm{A}}} t_{\mathrm{A}}+0,233-0,87 \delta
$$

dans laquelle $q_{n}$ (langley/mn) indique la consommation de la sphère noire, $t_{A}\left({ }^{\circ} \mathrm{C}\right)$ la température de l'air, $\delta$ (langley/mn) la différence de consommation entre l'une et l'autre des sphères.

Si l'on suppose que, au début ou à la fin de l'ablation, $\Delta$ et $t_{A}$ soient égaux à zéro, il résulte cette formule que la différence de consommation des deux sphères est $\delta=0,27$ langley/ mn. Cette valeur a une importance remarquable du moment qu'elle indique le point où commence la fusion; pour préciser, au-dessus de cette valeur, la fusion ne se produit pas; audessous, elle se produit.

Les frigorimètres Zanetti ont été installés à la Marmolada en 1960 à la cote 2100 , cinquante mètres au-dessus du lac de Fedaia. Ơn n'a effectué, au début, que des lectures journalières à 7 , 13 et 19 h. Etant donné la nature très variable du phénomène, on a transformé l'appareil en enregistreur et comme tel, après une laborieuse mise au point, il commencera à fonctionner l'hiver prochain.

Les observations qu'on a réalisées jusqu'à ce jour, toutefois, fournissent d'ores et déjà des confirmations intéressantes de la théorie exposée plus haut. Cela, on l'obtient en rapprochant Jes débits du canal d'amenée qui conduit au réservoir de la Fedaia l'eau d'ablation provenant du glacier, avec les mesures frigorimétriques. Le $\mathbf{5}$ avril, la mesure frigorimétrique a été inférieure à 0,27 langley/mn et, en effet, le canal a commencé à recevoir, à par- 
$\delta$

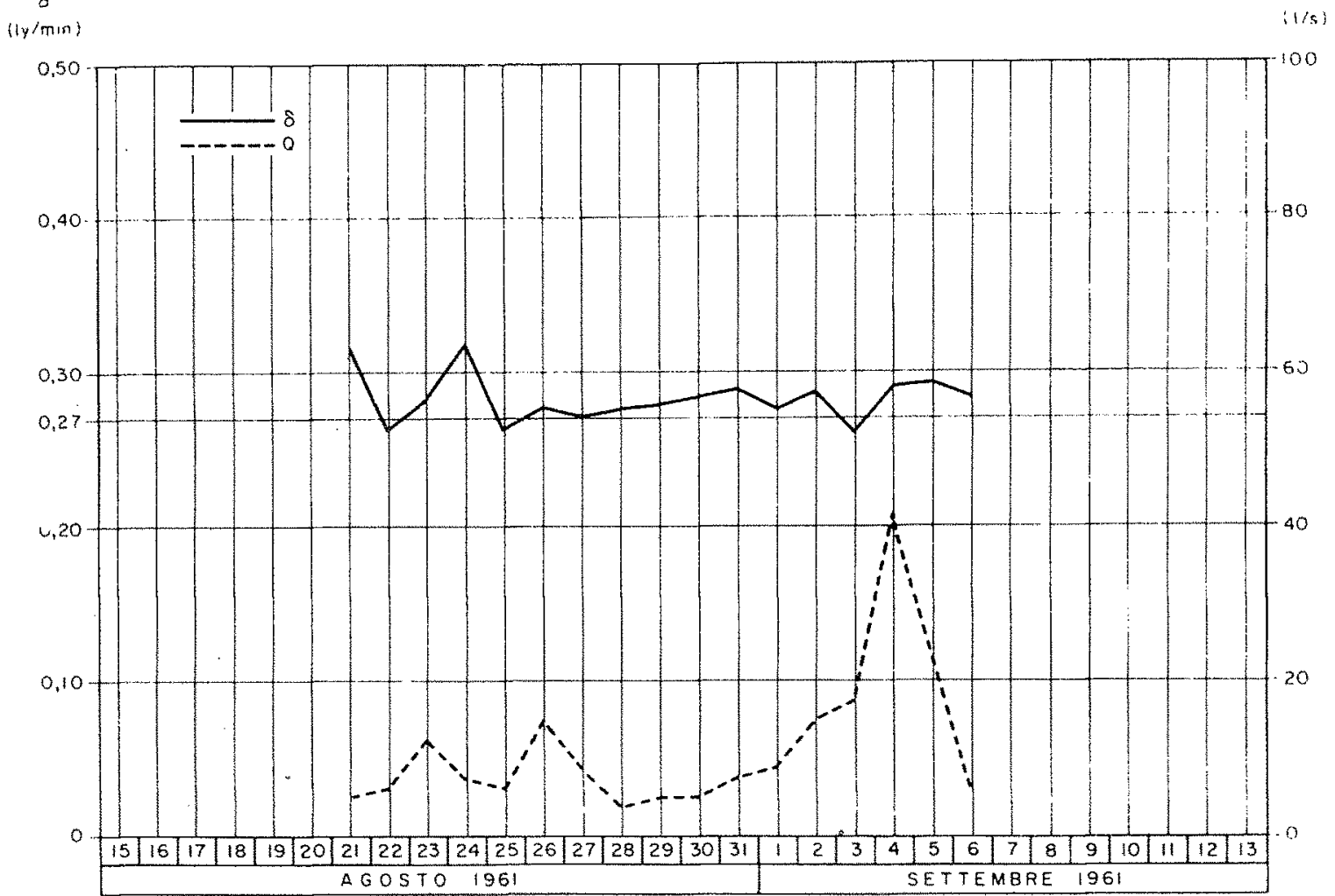

Fig. 4

Enregistrements frigorimétriques et débits d'ablation correspondant pendant la période du 15 aout au 15 septembre 1961 .

tir de cette époque, des débits d'ablation, même arec un certain décalage. Le 20 du même mois, la valeur de $\delta$ ayant dépassé 0,27 langley $/ \mathrm{mn}$, on a observé une cessation totale des débits, tandis que dans le mois de juin, la diminution de la valeur de $\delta$ a comporté une augmentation des débits. Une autre confirmation significative de la théorie exposée, est fournie (fig. 4) par les mesures effectuées à des intervalles de 5 nn dans la période qui va du 21 août au 5 septembre 1961 . Il a été possible d'obtenir séparément pour cette période, les valeurs de $\delta$ nocturnes, c'est-à-dire les valeurs négatives qui se sont vérifiées en l'absence de toute radiation soluire. Ces valeurs sont en bonne corrélation avec. les débits nocturnes afluant au canal; on signale notamment que, dans la nuit du 3 septembre, on a remarqué, parallèlement à une sensible diminution de la valeur absolue de $\delta$ négatif, une augmentation sensible, dans les débits mesurés.

Les conclusions qu'on vient d'exposer ont, pour le moment, un caractère d'orientation; elles sont toutefois assez intéressantes pour autoriser une poursuite des recherches dans ce domaine.

\section{4. - MESURES DESTINEES A DETERMINER LA CONSISTANCE ET LE COMPORTEMENT DU GLACIER}

C'est au mois de juillet 1952 qu'on a entrepris d'effectuer les observations et les recherches sur le glacier de la Marmolada, (fig. 5). A l'occasion de la visite du glacier faite par les membres de la Section de glaciologie de la Société Hydrotechnique de France, le Professeur P. Caloi, membre de l'Institut National de Géophysique, exécuta un sondage géosismique à la cote 2780 dans la localité dite Pian dei Fiacchi. D'après ce premier sondage, la profondeur de la glace atteignait, selon les points, de 40 à $50 \mathrm{~m}$.
Par la suite, à l'occasion de l'année géo-physique internationle, la SADE a effectué au mois de septembre 1959 et complété au cours de l'année 1960, un relevé géosismique. Vu les procédés employés et la présence probable de matériaux au fond (morainique glaciaire), les résultats qu'on a obtenus pour ce qui concerne le fond rocheux, peuvent être jugés exacts à $\pm 5 \%$.

Les résultats des relevés géosismiques, faits selon diverses sections, ont donné la possibilité de tracer des courbes de niveau concernant le 


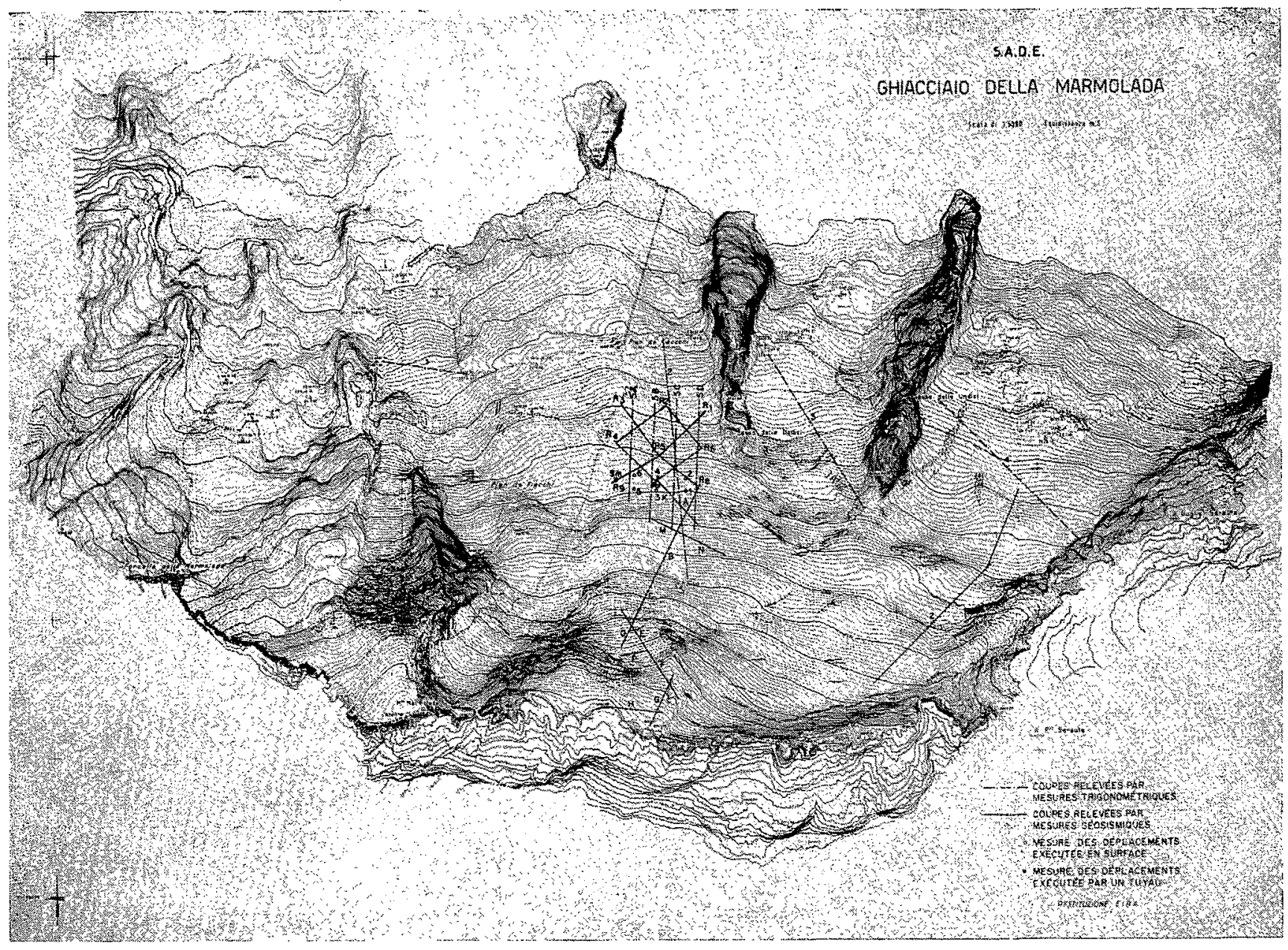

Fic. 5

Planimétrie du glacier de la Marmolada.

fond rocheux et la couche de glace qui les couvre. Les isohypses du fond rocheux et les lignes d'égale épaisseur de la glace ont été déterminées avec une approximation suffisante pour ce qui concerne la partie inférieure du relevé, alors que, pour la partie supérieure, on a adopté un procédé par interpolations en utilisant les différents éléments qu'on avait acquis de façon ou d'autre.

Les épaisseurs des différentes couches qu'on a rencontrées ont été mesurées par rapport aux vitesses de propagation respectives des ondes élastiques longitudinales. Celles-ci ont atteint $5,5 \mathrm{~km} / \mathrm{s}$ pour les matériaux calcaires,

\section{2,5 à $3,4 \mathrm{~km} / \mathrm{s}$}

pour la glace et $2 \mathrm{~km} / \mathrm{s}$ pour la neige. Il va sans dire qu'on n'a constaté aucun brusque passage de la neige à la glace, vu les différents degrés de transformation de la masse neigeuse; la limite conventionnelle de $2,0 \mathrm{~km} / \mathrm{s}$ s'est montrée suff- samment significative à l'égard des épaisseurs des accumulations de neige récente.

Les relevés ont fait ressortir le fait qu'a des cotes inférieures à 3050 , la couche superficielle de neige récente n'existe pas. Une autre remarque intéressante est celle qui concerne la diminution de la vitesse des ondes longitudinales vers le haut. Cela témoigne d'une augmentation progressive vers le bas de la rigidité de la glace, rigidité qui est due soit au poids, plus sensible, soit à la durée prolongée de l'action de refroidissement. Même l'absence de couche neigeuse dans le profil le plus élevé du relevé, c'est-à-dire à la cote $3225-3248$, absence due à une grosse avalanche qui s'est produite au cours de l'hiver 1958-1959, apparaît particulièrement significative à cet égard. La comparaison établie entre les différentes observations effectuées au cours de l'année 1958-1959 pour ce qui concerne la zone observée, permet de déterminer, avec plus de fondement, la diminution de la puissance de la glace qui se vérifie à l'intervalle d'une année. 
Cette diminution a été en moyenne de $1,40 \mathrm{~m}$ environ, avec des maximums de $3,70 \mathrm{~m}$.

D'après les éléments qu'on a repérés au cours des différentes observations, on peut conelure que la masse glaciaire de la Marmolada atteint actuellement $59631000 \mathrm{~m}^{3}$. De 1954 jusqu'à la fin de l'année 1959 , période pour laquelle on a des mesures fondées, on a constaté, en tenant compte des variations subies par le front aussi bien que de la diminution de la puissance, une diminution totale de la masse glaciaire de $18351000 \mathrm{~m}^{3}$ avec une valeur maximale de $3192000 \mathrm{~m}^{3}$ en 1954. Sur cette diminution cumulée, la variation du front influe pour un total de $1404000 \mathrm{~m}^{3}$, avec une valeur maximale de $315000 \mathrm{~m}^{3}$ en 1954 .

A titre d'orientation, on a pu conclure, en utilisant toutes les données disponibles, que la diminution de la masse glaciaire a été, à partir de ces dernières soixante dernières années, du même ordre que la diminution de la surface.

D'autre part, la vitesse des mouvements de la masse glaciaire a été aussi l'objet de recherches systématiques d'après lesquelles cette vitesse atteindrait en moyenne $1,10 \mathrm{~m}$ par an.

C'est avec le plus haut intérêt qu'on a suivi le parcours d'un tuyau de fer qu'on a enfoncé dans le glacier en 1954 et dont on observé les déplacements pendant cinq ans environ. Au cours de cette période, le tuyau s'est déplacé d'une façon linéaire en direction du nord pour environ $5 \mathrm{~m}$, mais sur des parcours progressivement décroissants. Dans les deux premières années, il y a eu, en effet, un déplacement de $2,33 \mathrm{~m}$ (parcours moyen annuel : 1,17); dans la deuxième période biennale, on a eu un parcours de 2,05 m (parcours moyen annuel : 1,03 ); enfin, dans la quatrième année, $0,92 \mathrm{~m}$ (fig. $6 \mathrm{a}$ ).

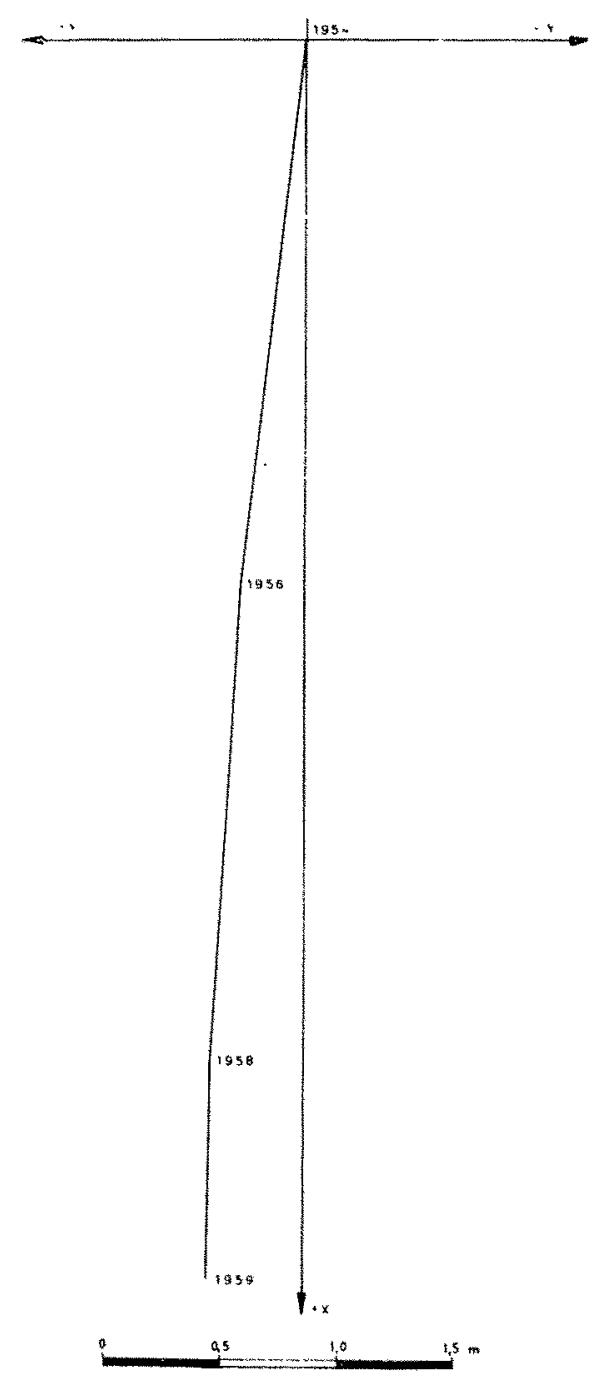

FIG. $6 a$

Déplacements d'un tuyau enfoncé dans la glace, mesurés durant la période $1954-1959$.
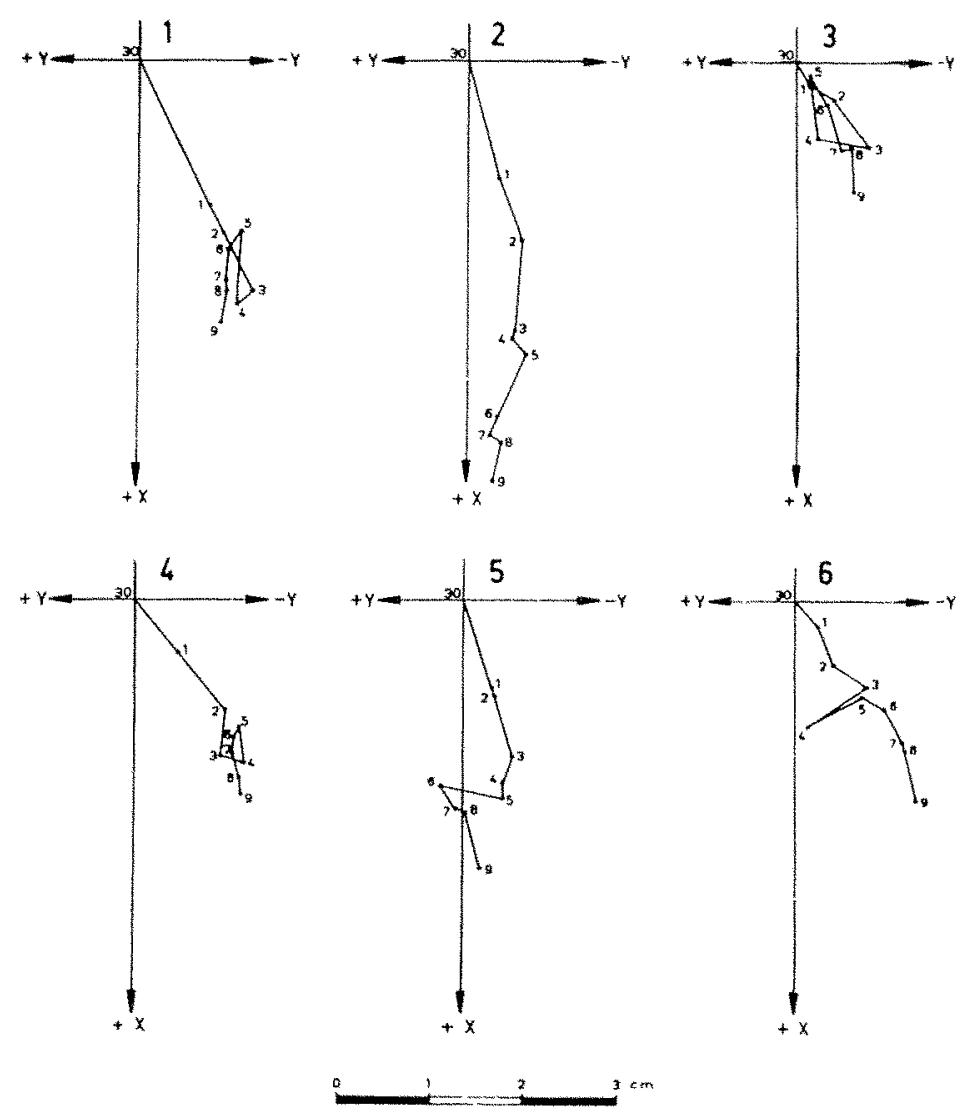

Fuc. $6 b$

Déplacements en surface de six points mesurés en 1959 . 
Ces observations nous ont engagés à entreprendre en 1959 des recherches systématiques dans six points (fig. $6 \mathrm{~b}$ ) dont les déplacements à la surface ont été mesurés au jour le jour et pendant dix jours de suite. Les résultats de ces mesures, rapportés dans un diagramme, montrent que les déplacements mêmes, excepté pour ce qui concerne le point 2, ont un cours assez mouvementé. D'ailleurs la brièveté des observations et l'époque oì elles ont été effectuées ne sauraient autoriser le recours à des extrapolations, bien qu'il soit intéressant de signaler qu'avec cette vitesse moyenne à la surface, on aurait des avancements moyens annuels de l'or- dre de ceux qu'on a obtenus avec le tuyau enfoncé dans la glace.

D'autres recherches sont en cours en vue de déterminer les apports qui alimentent directement le glacier dans les différentes époques de l'année, de même que le cours du processus d'ablation. On obtient cette détermination au moyen de tiges qu'on enfonce en nombre considérable sur toute la superficie du glacier.

Les observations et les résultats auxquels ont abouti les différentes recherches sont en cours d'élaboration. Elles seront présentées dans une publication que nous espérons pouvoir éditer bientôt.

\section{I S C U S I I N}

Président : M. Messines du Sourbiek

M. le Président remercie vivement, au nom de la S.H.F., M. Tonini d'avoir bien voulu faire ce long voyage depuis Venise pour nous exposer le résultat des travaux. effectués par les glaciologues italiens et par la Societa Adriatica di Elettricità au glacier de la Marmolada.

M. Ie Président remarque que ces études de bilan glaciaire se sont très grandement perfectionnées depuis le temps où notre section, qui venait de se former, procédait à des études semblables au glacier de Sarennes (1949). Il souligne l'originalité des études de M. Tonin I sur la radiation totale et, surtout, sur la séparation des différents éléments qui la constituent : cette analyse pcurra avoir une grande importance dans l'avenir pour la prévision des mouvements des glaciers et pour la détermination des apports dans les retenues hydroélectriques.

M. le Président se demande si la corrélation, constatée par M. Tonisi, entre la diminution de la masse glaciaire et celle de la surface du glacier de la Marmolada pendant les soixante dernières années, n'est pas une pure coïncidence, car une telle relation n'existe pas dans la plupart des glaciers en cuvette, notamment à Sarennes où l'on constate une énorme disparition de la masse glaciaire, malgré une très faible diminution de la surface. Cependant, M. le Président pense que la découverte de cette relation, qui paraît propre au glacier de la Marmolada, peut présenter un grand intérêt pour les prévisions d'apport de ce glacier.

M. Tonini répond que cette corrélation n'a pas été l'objet d'études détaillées, mais que les mesures de l'épaisseur et de l'étendue témoignent effectivement de l'existence d'une corrélation de ce type.

M. le Président s'étoune d'autre part de la faiblesse de la vitesse superficielle du glacier : $1 \mathrm{~m}$ à $1,50 \mathrm{~m}$ par an, soit $3 \mathrm{~mm}$ par jour en moyenne et $3 \mathrm{~cm}$ par jour en été, cela est très faible pour un tel glacier qui n'est pas un glacier de plateau.
M. Toninı précise que, vu le caractère particulier de la zone où les mesures ont été exécutées, on est amené à penser que la vitesse mesurée correspond à une valeur minimale.

M. Caspar demande quelques précisions sur les altitudes du glacier de la Marmolada et, également, sur celles des endroits où ont été faites les mesures d'équivalence neige/eau.

M. Tonnn précise ce qui suit :

Cote maximale du glacier (Punta Penia)... $3346,65 \mathrm{~m}$ Cote minimale du glacier ............ $2450 \mathrm{~m}$ Cote des stations de mesure de l'équivalent

en eau de la couche de neige......... $2000 \mathrm{~m}$

M. Tonini ajoute que le choix de la localité où sont installés les appareils a été conditionné par la nécessité de limiter la longueur du câble de liaison entre le compteur Geiger et les appareils calculateurs, pour. lesquels il faut aménager un local facilement accessible. Les recherches menées pour réaliser une liaison moyennant un pont-radio permettront dans quelque temps d'installer des points de mesure sur le glacier. Un prototype de pont-radio est en cours d'expérimentation à proximité d'un des appareils déjà existants.

M. Wouf demande si l'appareil servant à mesurer l'équivalent en eau de la couche de neige possède un circuit de contrôle de la chaîne électronique du compteur Geiger et comment l'on effectue l'étalonnage de cet appareil.

M. Tonini précise que, étant donné le type d'installation adopté, on n'a pas jugé nécessaire de procéder à l'étalonnage des appareils Geiger au moment de réaliser chacune des mesures. Un étalonnage est toutefois exécuté au début de chaque saison, en tenant compte de la radiation cosmique, dont cependant on n'est pas à même de mesurer les fluctuations constatées au cours de chacune des campagnes de mesure. 